\title{
Correction to: Pharmacokinetic and Pharmacodynamic Analyses of 5-Fluorouracil in East-Asian Patients with Nasopharyngeal Carcinoma
}

\author{
Yuxiang $\mathrm{Ma}^{1}$ - Yuehao $\mathrm{Lin}^{3} \cdot$ Benyan Zou $^{2}$ - Wanli Liu ${ }^{3}$ - Yang Zhang ${ }^{1}$ • \\ Liping Zhao ${ }^{4}$ - Yan Huang ${ }^{2}$ - Yunpeng Yang ${ }^{2}$ - Wenfeng Fang ${ }^{2}$ Yuanyuan Zhao ${ }^{2}$.

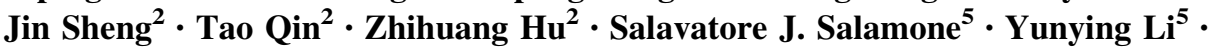 \\ Li Zhang ${ }^{2} \cdot$ Hongyun Zhao ${ }^{1}$
}

Published online: 26 December 2017

(C) Springer International Publishing AG, part of Springer Nature 2017

\section{Correction to: Clin Pharmacokinet (2016) 55(10): 1205-1216 https://doi.org/10.1007/s40262-016- 0395-2}

Page 1205, the author names and affiliations which previously read

Yuxiang $\mathrm{Ma}^{1}$, Yuehao $\mathrm{Lin}^{3}$, Benyan Zou ${ }^{2}$, Wanli Liu ${ }^{3}$, Yang Zhang ${ }^{1}$, Liping Zhao ${ }^{4}$, Yan Huang ${ }^{2}$, Yunpeng Yang ${ }^{2}$, Wenfang Fang ${ }^{2}$, Yuanyuan Zhao ${ }^{2}$, Jin Sheng ${ }^{2}$, Tao Qin ${ }^{2}$, Zhihuang $\mathrm{Hu}^{2}$, Li Zhang ${ }^{2}$, Hongyun Zhao ${ }^{1}$.

The original article can be found online at https://doi.org/10.1007/ s40262-016-0395-2.

Li Zhang

zhangli63@hotmail.com

$\triangle$ Hongyun Zhao

zhaohy@sysucc.org.cn

1 State Key Laboratory of Oncology in South China, Collaborative Innovation Center for Cancer Medicine, Clinical Trial Center, Sun Yat-Sen University Cancer Center, 651\# Dongfeng Road East, Guangzhou 510060, China

2 Department of Medical Oncology, State Key Laboratory of Oncology in South China, Collaborative Innovation Center for Cancer Medicine, Sun Yat-Sen University Cancer Center, 651\# Dongfeng Road East, Guangzhou 510060, China

3 Department of Medicine Laboratory, State Key Laboratory of Oncology in South China, Collaborative Innovation Center for Cancer Medicine, Sun Yat-Sen University Cancer Center, Guangzhou, China

4 Department of Medical Oncology, Yue Bei People's Hospital, Shaoguan, China

5 Saladax Biomedical, Inc., Bethlehem, PA, USA
1. State Key Laboratory of Oncology in South China, Collaborative Innovation Center for Cancer Medicine, Clinical Trial Center, Sun Yat-Sen University Cancer Center, 651\# Dongfeng Road East, Guangzhou, 510060, China.

2. Department of Medical Oncology, State Key Laboratory of Oncology in South China, Collaborative Innovation Center for Cancer Medicine, Sun Yat-Sen University Cancer Center, 651\# Dongfeng Road East, Guangzhou, 510060, China.

3. Department of Medicine Laboratory, State Key Laboratory of Oncology in South China, Collaborative Innovation Center for Cancer Medicine, Sun Yat-Sen University Cancer Center, Guangzhou, China.

4. Department of Medical Oncology, Yue Bei People's Hospital, Shaoguan, China.

\section{Should read}

Yuxiang $\mathrm{Ma}^{1}$, Yuehao $\mathrm{Lin}^{3}$, Benyan $\mathrm{Zou}^{2}$, Wanli $\mathrm{Liu}^{3}$, Yang Zhang ${ }^{1}$, Liping Zhao ${ }^{4}$, Yan Huang ${ }^{2}$, Yunpeng Yang ${ }^{2}$, Wenfeng Fang ${ }^{2}$, Yuanyuan Zhao' ${ }^{2}$ Jin Sheng ${ }^{2}$, Tao Qin ${ }^{2}$, Zhihuang $\mathrm{Hu}^{2}$, Salavatore J. Salamone ${ }^{5}$, Yunying $\mathrm{Li}^{5}, \mathrm{Li}$ Zhang $^{2}$, Hongyun Zhao ${ }^{1}$

1. State Key Laboratory of Oncology in South China, Collaborative Innovation Center for Cancer Medicine, Clinical Trial Center, Sun Yat-Sen University Cancer Center, 651\# Dongfeng Road East, Guangzhou 510060, China 
2. Department of Medical Oncology, State Key Laboratory of Oncology in South China, Collaborative Innovation Center for Cancer Medicine, Sun Yat-Sen University Cancer Center, 651\# Dongfeng Road East, Guangzhou 510060, China

3. Department of Medicine Laboratory, State Key Laboratory of Oncology in South China, Collaborative Innovation Center for Cancer Medicine, Sun Yat-Sen University Cancer Center, Guangzhou, China

4. Department of Medical Oncology, YueBei People's Hospital, Shaoguan, China

5. Saladax Biomedical, Inc., Bethlehem, PA, USA

Page 1214, Conflict of interest statement which previously read:

Yuxiang Ma, Yuehao Lin, Benyan Zoub, Wanli Liu, Yang Zhang, Liping Zhao, Yan Huang, Yunpeng Yang,
Wenfeng Fang, Yuanyuan Zhao, Jin Sheng, Tao Qin, Zhihuang Hu, Li Zhang, and Hongyun Zhao declare that they have no commercial or associative interests that represent a conflict of interest in connection with the work submitted.

\section{Should read}

Yuxiang Ma, Yuehao Lin, Benyan Zou, Wanli Liu, Yang Zhang, Liping Zhao, Yan Huang, Yunpeng Yang, Wenfeng Fang, Yuanyuan Zhao, Jin Sheng, Tao Qin, Zhihuang Hu, Li Zhang, and Hongyun Zhao declare that they have no commercial or associative interests that represent a conflict of interest in connection with the work submitted. Salavatore J. Salamone and Yunying Li, employees of Saladax Biomedical, Inc., Bethlehem, PA, USA, have stock in Saladax Biomedical. Saladax Biomedical developed the assay that was used in this study. 